\title{
BMJ Open Protocol for an open-label, single-arm trial of HIV pre-exposure prophylaxis (PrEP) among people at high risk of HIV infection: the NSW Demonstration Project PRELUDE
}

\author{
S Vaccher, ${ }^{1}$ A Grulich, ${ }^{1} \mathrm{~J}$ McAllister, ${ }^{2} \mathrm{D} \mathrm{J}$ Templeton, ${ }^{3} \mathrm{M}$ Bloch, ${ }^{4}$ A McNulty, ${ }^{5}$ \\ J Holden, ${ }^{6}$ I M Poynten, ${ }^{1}$ G Prestage, ${ }^{1,7}$ I Zablotska, ${ }^{1}$ on behalf of the PRELUDE \\ Study Team
}

To cite: Vaccher S, Grulich A, McAllister J, et al. Protocol for an open-label, single-arm trial of HIV pre-exposure prophylaxis (PrEP) among people at high risk of HIV infection: the NSW Demonstration Project PRELUDE. BMJ Open 2016;6:e012179.

doi:10.1136/bmjopen-2016012179

- Prepublication history and additional material is available. To view please visit the journal (http://dx.doi.org/ 10.1136/bmjopen-2016012179).

Received 6 April 2016 Revised 25 May 2016 Accepted 26 May 2016

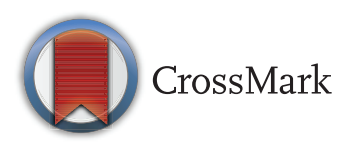

For numbered affiliations see end of article.

Correspondence to Iryna Zablotska; Izablotska@kirby.unsw.edu.au

\section{ABSTRACT}

Introduction: Despite a number of HIV prevention strategies, the number of new HIV infections remains high. In Australia, over three-quarters of new HIV diagnoses are in gay and bisexual men (GBM). Preexposure prophylaxis (PrEP) has been shown to be effective at preventing new HIV infections in several randomised trials. The PRELUDE study aims to evaluate the implementation of PrEP in healthcare settings in New South Wales (NSW), Australia, among a sample of high-risk adults.

Methods and analysis: PRELUDE is an ongoing open-label, single-arm demonstration project, conducted in public and private clinics across NSW, Australia. Enrolment began in November 2014. The study is designed for 300 high-risk participantsmainly GBM and heterosexual women. Participants receive daily oral PrEP, composed of emtricitabine (FTC) and tenofovir disoproxil fumarate (TDF), for up to 2.5 years. Quarterly study visits include testing for HIV and sexually transmitted infections (STIs), assessment of ongoing eligibility and side effects, and self-reported adherence. Following each study visit, online behavioural surveys are administered to collect information on medication adherence, risk behaviours and attitudes. Blood samples will be collected in a subset of patients 1, 6 and 12 months after PrEP initiation to measure FTC/TDF concentrations. Analyses using longitudinal regression models will focus on feasibility, adherence, safety, tolerability and effects of PrEP on behaviour. This study will inform PrEP policy and guide the implementation of PrEP in Australia in people at high risk of HIV.

Ethics and dissemination: The study will be conducted in accordance with the Declaration of Helsinki. All patients will provide written informed consent prior to participation in the study. Publications relating to each of the primary end points will be gradually released after 12 months of follow-up is complete.

Trial registration number: NCT02206555; Pre-results.

\section{Strengths and limitations of this study}

- This is an open-label trial which will better reflect 'real-world' behavioural, attitudinal and adherence practices, in comparison with previous randomised controlled trials.

- The study is open to groups of people excluded from previous PrEP research, including women trying to conceive naturally, and transgender men and women.

- This is a relatively small sample with no comparison group, but detailed data collection will allow greater insights into the study population and the effects of PrEP use in 'real-world' circumstances.

\section{BACKGROUND}

According to the Joint United Nations Programme on HIV/AIDS (UNAIDS), there were 2.3 million new HIV infections globally in 2012, bringing the total number of people living with HIV to $\sim 35.3$ million. ${ }^{1}$ In Australia, HIV diagnoses have remained stable over the past 3 years, with the majority of new infections occurring in gay and bisexual men $(\mathrm{GBM}) .^{2}$ The efficacy of preexposure prophylaxis (PrEP) in preventing HIV in heterosexuals and GBM has been demonstrated in several randomised controlled trials. ${ }^{3-6}$ The Pre-exposure Prophylaxis Initiative (iPrEx) study found a $92 \%$ (95\% CI $40 \%$ to $99 \%$ ) reduction in the relative risk of $\mathrm{HIV}$ infection among men who have sex with men and transgender women who had detectable levels of study drug in plasma samples, and were thus highly adherent to daily PrEP, in comparison with people who had no detectable PrEP in their blood samples. ${ }^{4}$ However, it must be 
noted that this risk reduction estimation comes from a case-control study within the active arm of a randomised controlled trial, thus subjecting the results to confounding and bias. Medication adherence was closely correlated with efficacy, with only $51 \%$ of the cohort having detectable blood levels of study drug. The overall relative risk reduction among the entire cohort was $44 \%$ (95\% CI $15 \%$ to $63 \%$ ). Other clinical trials and demonstration studies have confirmed a strong relationship between medication adherence and PrEP efficacy, ${ }^{78}$ with much higher levels of PrEP effectiveness found in the preexposure prophylaxis to prevent the acquisition of HIV-1 infection (PROUD) (HIV risk reduction 86\%, 90\% CI $64 \%$ to $96 \%)^{9}$ and France Recherche Nord et Sud SidaHIV et Hépatites Intervention Préventive de l'Exposition aux Risques avec et pour les Gays (ANRS IPERGAY) (HIV risk reduction $86 \%, 95 \%$ CI $40 \%$ to $98 \%)^{10}$ studies than the earlier clinical trials.

The study medication, a fixed-dose, coformulation of $200 \mathrm{mg}$ emtricitabine (FTC) and $300 \mathrm{mg}$ tenofovir disoproxil fumarate (TDF), was approved by the US Food and Drug Administration in July 2012 for HIV prevention. ${ }^{11}$ Several other countries have followed suit, including France, Peru, South Africa, and most recently, Australia. Recent WHO guidelines recommend PrEP for all individuals at substantial risk of acquiring HIV, along with condom usage and other risk reduction practices. ${ }^{12}$ These guidelines also recommend the establishment of implementation projects to examine the feasibility of PrEP in a variety of settings.

For these reasons, the New South Wales (NSW) Ministry of Health has supported the establishment of the PrEP demonstration project PRELUDE, which aims to evaluate the implementation of evidence-based provision of PrEP in healthcare settings in NSW among a sample of individuals at high risk of acquiring HIV.

\section{Research questions}

The primary objectives of the study are to assess:

1. The feasibility of the process of PrEP delivery in public and private healthcare settings in NSW.

2. The acceptability of PrEP among high-risk adults invited to participate in the PRELUDE study.

3. Factors associated with longer term PrEP use, including:

A. Adherence to PrEP among HIV-negative individuals.

B. Experiences and perceptions of side effects associated with PrEP use, and their impact on adherence.

C. Behavioural effects of, and attitudes to, PrEP use. Additionally, the study has two exploratory objectives:

1. Document the cost of PrEP provision in a NSW healthcare setting, including the feasibility, funding and potential copayment schemes in the likelihood of future PrEP provision, following completion of this project.

2. Assess biological markers of adherence to FTC/TDF and compare them with participant self-reported adherence.
METHODS/DESIGN

\section{Summary of study design}

PRELUDE is a prospective, open-label, single-arm, multicentred study, coordinated by the Kirby Institute at UNSW Australia. Participants are HIV-negative GBM and heterosexual women at high risk of future HIV acquisition, taking daily FTC/TDF PrEP. At each study visit, participants are assessed for ongoing eligibility (box 1) and willingness to continue taking daily PrEP, receive testing for HIV, sexually transmitted infections (STIs), and renal and liver function, undergo assessment of adverse events and medication adherence by self-report, discuss risk reduction strategies, and receive condoms and a 30-day supply of PrEP. Patients are also asked to complete a self-administered online survey about attitudes, behaviours and adherence to PrEP following each study visit. Enrolment started in November 2014, and the study is designed to follow at least 300 participants.

\section{Study extension}

While the initial study was conceived to be 1 year long, resulting in 300 person-years (PYs) of follow-up in 300 individuals, an additional 18 month supply of FTC/TDF has been approved by Gilead. This means that all 300 patients enrolled in the study will now be followed for a total of 2.5 years, resulting in a total of 750 PYs of follow-up. These details are included in the latest protocol amendment, V.4.0, dated 30 September 2015,

Box 1 Behavioural eligibility criteria for prescribing pre-exposure prophylaxis (PrEP) for gay/homosexual men

High-risk patients (recommend prescribing daily PrEP) if a patient acknowledges:

Being likely to have multiple events of unprotected anal intercourse (UAI), ( \pm sharing injecting drug use, IDU), in the next 3 months (sustained risk);

AND also have any of the following:

Regular sexual partner of an HIV-infected man with whom condoms were not consistently used in the last 3 months (HIV-positive partner is not on treatment and/or has detectable viral load);

At least one episode of receptive UAI with any casual HIV-infected male partner or a male partner of unknown HIV status in the last 3 months;

Rectal gonorrhoea or chlamydia diagnosis during the last 3 months or at screening;

Methamphetamine use in the last 3 months.

Medium-risk patients (consider prescribing daily PrEP) if a patient acknowledges:

Being likely to have multiple events of UAI ( \pm sharing IDU) in the next 3 months (indicating sustained risk);

AND also have any of the following:

More than one episode of anal intercourse in the last 3 months when proper condom use was not achieved (eg, condoms slipped off or broke);

If client is uncircumcised and reports more than one episode of insertive UAI in the last 3 months where the serostatus of partner was not known or was HIV positive and not on treatment. 
which the study procedures and details in this paper are based on.

\section{Sample size}

As the PRELUDE study was designed as a demonstration project, the sample size was not determined by power calculations, but was informed by currently available evidence, expressions of interest for PrEP and the number of participants deemed feasible to enrol through clinics in NSW. Given the drug availability and funding provided, a sample size of 300 individuals was chosen, with the possibility of enrolling up to 30 heterosexuals $(10 \%$ of the total study cohort).

\section{Eligibility}

The full list of the study inclusion and exclusion criteria is presented in table 1. In summary, eligible patients are aged over 18 years, documented to be HIV-negative at enrolment, willing to undergo all study procedures, and at medium-to-high risk of acquiring HIV, as defined by the behavioural eligibility criteria (box 1 ). These criteria were developed by an expert group of clinicians, researchers and community representatives to guide the PRELUDE study, and were incorporated into the Interim NSW PrEP guidelines, ${ }^{13}$ which were based on the US Centers for Disease Control and Prevention (CDC) Interim Guidance on PrEP. ${ }^{14}$ The PRELUDE study was then established to provide PrEP to high-HIV-risk individuals, evaluate the NSW interim guidelines and inform the development of the Australian National PrEP Guidelines. ${ }^{15}$

The behavioural eligibility criteria took into account risk factors known to be associated with the highest incidence of HIV infection among Australian GBM. ${ }^{16-19}$ This ensured that PRELUDE targeted individuals who were at the highest risk of acquiring HIV and would thus derive the greatest benefit from free access to PrEP. A prescreening eligibility assessment was developed to assess if patients were eligible for PrEP based on the aforementioned criteria.

\section{Clinical sites and enrolment of participants}

The study roll-out was conducted in two stages. During stage I, participants were enrolled in four large public HIV and sexual health services in NSW (RPA Sexual Health Clinic, Sydney Sexual Health Centre, St Vincent's Hospital HIV, Immunology and Infectious Diseases Unit and Western Sydney Sexual Health Centre). In stage II, the model of care developed by these services was replicated in a further two public HIV and sexual health clinics (Clinic 16 at Royal North Shore Hospital and Newcastle Sexual Health Service) and two private primary care clinics (Holdsworth House Medical Practice and Taylor Square Private Clinic).

Participant recruitment has been undertaken by a variety of methods. The majority of participants are already attending the participating clinics, are identified as being at high risk of HIV by staff, and are provided with information about the PRELUDE study. Other patients are referred from s100 prescribers, which is a network of clinicians that are able to prescribe antiretrovirals and other highly specialised drugs. Some patients also self-refer, learning about the study through the PRELUDE website, from friends who were participating or by word of mouth. All participants are asked by clinicians to sign a written informed consent form prior to eligibility assessment, and another before enrolling in the study. A copy of these forms can be viewed in the appendix. Site investigators are permitted to discontinue participants if they no longer meet the study eligibility criteria (table 1).

Table 1 Inclusion and exclusion criteria for participants in the PRELUDE study

\begin{tabular}{|c|c|}
\hline Inclusion criteria & Exclusion criteria \\
\hline $\begin{array}{l}\text { HIV negative at enrolment, documented by a } \\
\text { negative HIV test }<7 \text { days prior to initiating PrEP } \\
\text { At high and ongoing risk of acquiring HIV infection } \\
\text { through sexual exposure } \\
\text { Aged } 18 \text { years or over } \\
\text { Currently residing in NSW, or visiting NSW with } \\
\text { sufficient frequency for participation } \\
\text { Medicare eligible } \\
\text { Willing and able to provide informed consent } \\
\text { Willing and able to take part in all required study } \\
\text { procedures } \\
\text { Proficient in written and spoken English }\end{array}$ & $\begin{array}{l}\text { HIV-1 infected, or has symptoms consistent with acute viral infection } \\
\text { Having an estimated glomerular filtration rate of }<60 \mathrm{~mL} / \mathrm{min} \\
\text { Concurrently taking a nephrotoxic agent } \\
\text { Having or developing clinical symptoms suggestive of lactic acidosis } \\
\text { or pronounced hepatotoxicity } \\
\text { Allergic to tenofovir disoproxil fumarate and/or emtricitabine } \\
\text { Concurrently taking prescribed products which contain tenofovir } \\
\text { disoproxil fumarate or emtricitabine } \\
\text { Mental health issues, memory loss, cognitive impairment or other } \\
\text { intellectual disability that may compromise participant safety or } \\
\text { regimen adherence } \\
\text { Conditions which may compromise a participant's retention in the } \\
\text { study } \\
\text { Unwilling to adhere to any of the required procedures } \\
\text { Currently breastfeeding* }\end{array}$ \\
\hline
\end{tabular}

*Safety for infants exposed to FTC/TDF PrEP has not yet been established. No harm has been reported for infants exposed during pregnancy and as such, pregnancy (or plans to become pregnant) is not an exclusion criterion for this study. However, women should learn about the risks and benefits of using PrEP during pregnancy prior to entering the study.

FTC, emtricitabine; NSW, New South Wales; PrEP, pre-exposure prophylaxis; TDF, tenofovir disoproxil fumarate. 


\section{Study visits}

Table 2 shows the study assessment procedures. The 30-month study plan consists of:

- visit ' 0 ', to assess eligibility;

- enrolment/baseline visit;

- 11 medication follow-up visits;

- follow-up visit 3 months post medication discontinuation; and

- a behavioural survey to be completed following each study visit, as well as 3 and 6 months after study completion or discontinuation.

Prior to enrolment, each patient undergoes testing for HIV, chlamydia, gonorrhoea and syphilis, as well as for hepatitis B (vaccination was offered if antibody negative). At each study visit, ongoing eligibility is confirmed, participants are asked about adverse events and medication adherence, are screened for HIV and other STIs, undergo laboratory evaluation of renal and liver function, and women of childbearing age undergo a pregnancy test. In addition, each participant is required to complete an online survey of behaviour, attitudes and adherence. The baseline study visit includes a complete medical history, while subsequent visits evaluate any changes in health status and medication-specific side effects. Discussions about HIV and STI risk reduction, information about medication adherence and risk reduction strategies, and condoms are offered to participants at every visit.

\section{Behavioural surveys}

The research team has extensive experience in developing behavioural surveys and has established links with the GBM community, which has high levels of altruism and interest in HIV research. To ensure adequate completion of behavioural surveys, clinicians will remind participants of the importance of prompt survey completion at each study visit. If a survey remains incomplete 3 days after a study visit, participants will receive two automated email reminders, 1 week apart, followed by clinic intervention using phone or Short Message Service (SMS) reminders.

\section{Blood collection substudy}

A convenience sample of $\sim 100$ patients enrolled in the three largest study clinics (Holdsworth House Medical Practice, Sydney Sexual Health Centre and St Vincent's Hospital HIV, Immunology and Infectious Diseases Unit) has also been asked to participate in a blood collection substudy. These clinics were chosen as they are located closest to the storage facility, St Vincent's Centre for Applied Medical Research, thus reducing the need for couriering and storage. Consecutive consenting participants at each site will be enrolled until there are at least 100 participants in the substudy. All sites have sufficient numbers of participants for enrolment in the substudy, as well as previous experience with blood sample collection for research studies. Plasma and peripheral blood mononuclear cells (PBMCs) are isolated from blood samples taken at months 1, 6 and 12, and are stored for assessment of TDF and FTC concentrations in blood, thus enabling an objective measure of medication adherence and comparison to self-reported adherence measures. These patients are also asked if they are willing to provide a separate informed consent for an optional serum sample (6 mL taken at months 1, 6 and 12), which will be collected and stored for future research. This consent form can also be viewed in the appendix. Analysis of blood samples for TDF/FTC concentrations is undertaken by the Departments of Pathology and Medicine laboratory at Johns Hopkins University School of Medicine, Baltimore, USA, in accordance with standard operating procedures.

\section{End points}

In line with the primary objectives of the study, there are five primary end points that will be pursued to determine the effectiveness and appropriateness of PrEP implementation in NSW (box 2). These can be divided into individual-level end points (safety, adherence and behavioural effects) and community-level end points (feasibility and acceptability).

Adherence to PrEP is a key focus of the PRELUDE study and is being assessed through four different methods: (1) through plasma and PBMC drug concentrations; (2) pharmacy pill-dispensing records; (3) patient self-report to clinician during clinic visits and (4) patient self-reported adherence in online surveys, as well as questions about barriers to adherence or breaks from taking the study medication. Correlation between these measures will be assessed at months 1, 6 and 12 (time points when blood collection occurs), and changing patterns of adherence throughout the study will be examined.

Another specific focus of this study is to investigate long-term behavioural changes in patients taking PrEP. It has been suggested that PrEP use may encourage users to forego other methods of risk reduction, such as condom use, because they believe PrEP is protecting them against HIV. ${ }^{20}$ Previous studies have shown mixed results, ${ }^{21-25}$ but the overwhelming evidence is that such changes in risk reduction behaviour have not been observed in PrEP cohorts to date.

The list of behavioural end points being investigated in the PRELUDE study can be viewed in box 2. In order to measure any changes in behaviour in our sample, data on a variety of risk markers such as STIs, number of HIV-positive casual partners and number of episodes of condomless anal intercourse (CLAI) will be collected regularly throughout the study.

\section{Adverse events}

Serious adverse events are life-threatening events, including HIV seroconversions, that must be reported to the 
Table 2 Summary of the PRELUDE study procedures

\begin{tabular}{|c|c|c|c|c|c|c|c|c|c|c|c|}
\hline $\begin{array}{l}\text { Data } \\
\text { collection } \\
\text { round }\end{array}$ & $\begin{array}{l}\text { Screening } \\
\text { Visit 0* }\end{array}$ & $\begin{array}{l}\text { Baseline } \\
\text { Visit } 1\end{array}$ & $\begin{array}{l}\text { Follow-up } \\
1 \\
\text { Visit } 2\end{array}$ & $\begin{array}{l}\text { Follow-up } \\
2 \\
\text { Visit } 3\end{array}$ & $\begin{array}{l}\text { Follow-up } \\
3 \\
\text { Visit } 4\end{array}$ & $\begin{array}{l}\text { Follow-up } \\
4 \\
\text { Visit } 5\end{array}$ & $\begin{array}{l}\text { Follow-up } \\
5 \\
\text { Visit } 6\end{array}$ & $\begin{array}{l}\text { Follow-ups } \\
6-10 \\
\text { Visits 7-11 }\end{array}$ & $\begin{array}{l}\text { Follow-up } 11 \\
\text { Visit } 12\end{array}$ & $\begin{array}{l}\text { Post-PrEP } \\
\text { assessment }\end{array}$ & $\begin{array}{l}\text { Data } \\
\text { collection } \\
\text { round }\end{array}$ \\
\hline Timeline & Week 2 & 0 & Month 1 & Month 3 & Month 6 & Month 9 & Month 12 & $\begin{array}{l}\text { Months } \\
15-27\end{array}$ & $\begin{array}{l}\text { Month 30/ } \\
\text { discontinuation }\end{array}$ & $\begin{array}{l}3 \text { months post } \\
\text { discontinuation }\end{array}$ & $\begin{array}{l}6 \text { months post } \\
\text { discontinuation }\end{array}$ \\
\hline $\begin{array}{l}\text { Review } \\
\text { eligibility }\end{array}$ & $x$ & $x$ & $x$ & $x$ & $x$ & $x$ & $x$ & $x$ & & & \\
\hline $\begin{array}{l}\text { Medical } \\
\text { history }\end{array}$ & $x$ & $x$ & & & & & & & & & \\
\hline Lab tests & $x$ & $x$ & $x \dagger$ & $x$ & $x \dagger$ & $x$ & $x \dagger$ & $x$ & $x$ & $x$ & \\
\hline $\begin{array}{l}\text { Dispense } \\
\text { medication }\end{array}$ & & $x$ & $x$ & $x$ & $x$ & $x$ & $x$ & $x$ & & & \\
\hline $\begin{array}{l}\text { STI } \\
\text { testing }\end{array}$ & $x \S$ & $x$ & & $x$ & $x$ & $x$ & $x$ & $x$ & $x$ & $x$ & \\
\hline HIV testing & $x$ & $x$ & $x$ & $x$ & $x$ & $x$ & $\mathrm{x}$ & $x$ & $x$ & $x \|$ & $x \|$ \\
\hline Survey & $x$ & $x$ & $x$ & $x$ & $x$ & $x$ & $x$ & $x$ & $x$ & $x$ & $x$ \\
\hline
\end{tabular}

*Visits 0 and 1 may be combined.

†Blood collected for plasma/PBMCs/serum at months 1, 6 and 12

fObtained per standard of care.

§Hepatitis B status obtained prior to baseline.

ПHIV status post-PrEP discontinuation may be obtained via data linkage.

PBMCs, peripheral blood mononuclear cells; PrEP, pre-exposure prophylaxis; STI, sexually transmitted infection. 


\section{Box 2 Primary end points of the PRELUDE study}

- Feasibility of the process of pre-exposure prophylaxis (PrEP) delivery in a healthcare setting in New South Wales (NSW)

- Accrual of 300 person-years of PrEP;

- Seroconversion-free time on PrEP;

- Retention rates among participants enrolled in the study.

- Acceptability

- Eligibility for PrEP among individuals entering the screening process;

- Among individuals found eligible to participate in the study, proportion who provide their consent to participate.

- Adherence

- Time to PrEP discontinuation;

- Number, proportion and patterns of prescribed doses taken and missed (estimated from self-report and dispensing records)

- Safety and side effects

- Incidence of HIV seroconversion among study participants during the study, and in the 6 months following PrEP discontinuation;

- Serious adverse events;

- Any adverse events, and in particular those leading to the interruption or discontinuation of the study product.

- Behavioural effects of PrEP use

- Level of awareness and perception of HIV risk;

- Participant self-reported frequency of sexual activity and condom use;

- Participant self-reported likelihood of condom use after discontinuation of PrEP;

- New gonorrhoea or chlamydia infections;

- Pregnancy.

sponsor within one business day, and the lead ethics committee within 72 hours. All events will be judged by a medical officer as related or unrelated to the study drugs, and will be subsequently reported to Gilead. All pregnancies in women taking PrEP will also need to be reported to Gilead. No prespecified adverse event data are being collected; however, testing for STIs and renal and hepatic function will occur at each study visit. Clinicians will ask participants to report any other adverse events or untoward medical occurrences at each quarterly visit, or at an additional visit if they present to the clinic between study visits, and these events will be captured by the clinical data management system. All participants reporting an adverse event will be followed clinically until it is resolved or stabilised.

\section{HIV seroconversions}

Participants are tested for HIV and asked about any symptoms of acute seroconversion illness at each clinic visit. In addition, data linkage to the NSW HIV and STI registry, maintained by the NSW Ministry of Health, will also allow identification of any PRELUDE participant who seroconverts within 6 months of completing the study. Participants who acquire HIV during the study will cease PrEP immediately and be managed according to the Australian national treatment guidelines. ${ }^{26}$

\section{Data collection}

Data collection occurs by a variety of methods. Paper-based prescreening forms are completed by the participant or practitioner in the clinic during the screening visit. Pharmacy data (pill counts and dispensing records) are also recorded on paper. All other data collection is completed by clinic staff using electronic case report forms on OpenClinica, an open-source clinical trial software for electronic data capture and clinical data management (2015 OpenClinica, LLC, developed by Isovera, Inc). This includes results from laboratory tests, information on adverse events and concomitant medication use, and participant self-reported adherence to clinicians. OpenClinica Enterprise complies with US 21 Code of Federal Regulations Part 11, Electronic Records; Electronic Signatures. Roles and access are controlled, electronic signatures are enabled, protected health information is non-identifiable and the database is SSL encrypted. Regular monitoring will occur to ensure completeness and quality of the clinical data collected in the PRELUDE study.

After each study visit, participants are emailed an individual link to the online questionnaire, which contains their unique study identification number embedded within it. The baseline questionnaire contains social and demographic questions that are not repeated in subsequent surveys; all follow-up surveys are composed of the same set of questions, with skip logic dependent on the participants' responses. All survey data are collected using an online survey design and implementation platform SurveyGizmo (Boulder, Colorado, USA). Data entered into the online forms use the participants' study identification number, with no identifying details recorded. SurveyGizmo is compliant and certified under the Health Insurance Portability and Accountability Act (HIPAA) (US guidelines for the handling of medical information) and Safe Harbor (European Union privacy protection standards) programmes. The final data set will be deidentified and stored securely at the Kirby Institute.

\section{Data analysis}

All participants are given a unique study identification number that is used across all data platforms to allow linkage of clinical and behavioural databases, as well as to follow each participant longitudinally through the study. Future analyses using PRELUDE study data will focus on HIV seroconversions, adherence to PrEP and changes to risk behaviours during the course of the study, as well as investigating retention rates and adverse events among participants.

Statistical analysis of sexual practices, adherence to study medication and risk of HIV seroconversion will be conducted using longitudinal regression models with time-varying exposures in STATA (StataCorp, College Station, Texas, USA). Demographic information obtained from surveys will be analysed using descriptive statistics, while more complex behavioural and 
attitudinal data will be analysed using cross-sectional and longitudinal analysis. All data submitted to the study will be collated prior to analysis, and the effects of the missing data will be investigated.

\section{Ethics and dissemination}

The PRELUDE study has been designed and conducted by a research team at the Kirby Institute, University of New South Wales, Australia (Protocol Chair Dr Iryna Zablotska). The study protocol was approved by St Vincent's Hospital Human Research Ethics Committee (HREC) in Sydney, NSW (protocol number HEPP 1403; identification number HREC/14/SVH/130), and registered under ClinicalTrials.gov (identifying number NCT02206555). Site-specific assessment was obtained from each clinic prior to starting enrolment. All study participants provided written informed consent before undergoing any of the study procedures. The study will be conducted in accordance with the Declaration of Helsinki and European Committee for Proprietary Medicinal Products (CPMP)/ International Council for Harmonisation of Technical Requirements for Pharmaceuticals for Human Use (ICH) Guidelines for Good Clinical Practice. Meetings of the Protocol Steering Committee (PSC) and Data Safety and Monitoring Board (DSMB) will be conducted at least annually, both of which include representatives of community HIV organisations. Interim analysis occurred following enrolment of the first 100 patients, with results being reported back to the PSC and DSMB, as well as participants, the NSW Ministry of Health and relevant community organisations. The primary outcome paper about HIV seroconversions during the study will be published on completion of 12 months of follow-up of all participants. Publications of the other primary study end points (box 2) are scheduled in HIV-field peer-reviewed journals following the dissemination of the primary end point paper. Findings will also be presented at key national and international HIV conferences, as well as presented at community forums and HIV and public health sector events for broad dissemination of study outcomes.

\section{DISCUSSION}

Despite numerous completed and ongoing PrEP studies throughout the world, the PRELUDE study has specific value for two reasons. First, this project has been developed in conjunction with, and has informed the development of, the Australian National PrEP Prescribing Guidelines, ${ }^{15}$ and seeks to target populations at highest risk of HIV acquisition. Australia is the third country worldwide to develop National PrEP Prescribing Guidelines, after the $\mathrm{USA}^{14}$ and South Africa. ${ }^{27}$ Thus, the findings from the PRELUDE study will play a vital role in the evaluation and further development of these guidelines, particularly now that PrEP is approved for sale within Australia as HIV prophylaxis.
Based on the PRELUDE eligibility criteria and previous Australian research, ${ }^{16}$ patients in this cohort are expected to be at particularly high risk of acquiring HIV in the future. The cost of FTC/TDF PrEP remains high in Australia ( $\$$ A13 500 (US\$9645) per annum for an off-label prescription ${ }^{28}$ ); thus, access to PrEP under the universal Australian healthcare system will only occur if it is shown to be cost-effective. Previous studies from overseas $^{29}{ }^{30}$ and Australia ${ }^{31}$ have found PrEP to be costeffective if targeted to individuals at high risk of HIV.

This study is not without some limitations. First, it has enrolled a relatively small sample of 300 individuals, with follow-up restricted to a maximum of 2.5 years, so there is limited potential to detect long-term trends. Despite this, frequent detailed data collection, particularly on behavioural practices and STI testing and diagnosis, and procedures to maintain high rates of follow-up will ensure that a high-quality and complete data set is available for analysis. This is a one-arm study and there is no comparison group, but as PRELUDE is a feasibility and implementation study, this group is not necessary. We anticipate that only a small number of heterosexuals will be enrolled in the study, so we will not be able to conduct quantitative analysis on this group, but given the nature of the HIV epidemic in Australia being predominantly in GBM, this group is our primary focus for data analysis.

In conclusion, the PRELUDE study is a demonstration project designed to establish and evaluate an Australian model of prescribing oral PrEP to individuals at high risk of HIV infection. Given the planned extensive data collection and analysis, this study will provide crucial information on PrEP implementation in Australia and the impacts of PrEP use on the wider community. It will also play a major role in developing and assessing the Australian National PrEP Guidelines, resulting in optimal access to PrEP for those who need it most.

\section{Author affiliations}

${ }^{1}$ Kirby Institute, UNSW Australia, Kensington, New South Wales, Australia ${ }^{2}$ St Vincent's Hospital, Darlinghurst, New South Wales, Australia ${ }^{3}$ RPA Sexual Health, Sydney Local Health District, Camperdown, New South Wales, Australia

${ }^{4}$ Holdsworth House Medical Centre, Darlinghurst, New South Wales, Australia ${ }^{5}$ Sydney Sexual Health Centre, Sydney, New South Wales, Australia ${ }^{6}$ NSW Ministry of Health, North Sydney, New South Wales, Australia ${ }^{7}$ Australian Research Centre in Sex, Health and Society, La Trobe University, Melbourne, Victoria, Australia

Acknowledgements This study was funded by the NSW Ministry of Health. Study medication was provided by Gilead. The authors would also like to thank all the participants in the study, the study staff, clinical and laboratory staff, and staff in gay and HIV community organisations for supporting this research.

Collaborators The PRELUDE Study Team are Mark Bloch, Andrew Carr, Ching (Yvonne) Cheung (study clinical coordinator), Rosalind Foster, Chris Gianacas (research assistant-clinical data management), Andrew Grulich, Rebecca Guy, Martin Holt, John Kaldor, Brent Mackie, Kenneth Mayer, John McAllister, Anna McNulty, Dean Murphy, Catriona Ooi, Cathy Pell, Mary Poynten, Garrett Prestage, Nathan Ryder, David Templeton, Stefanie Vaccher 
(research assistant- behavioural data management), John de Wit, Edwina Wright, Iryna Zablotska (Chief Investigator and Protocol Chair). Additional collaborators are AIDS Council of NSW (ACON), Sydney, Australia; Centre for Social Research in Health, UNSW Australia, Sydney, Australia; Clinical Pharmacology Analytical Lab, The Johns Hopkins University, Baltimore MD, USA; Positive Life NSW, Sydney, Australia; St Vincent's Centre for Applied Medical Research (AMR), Sydney, Australia; Sydpath Central Laboratory, St Vincent's Hospital, Sydney, Australia.

Contributors IZ, AG, JM, DJT, IMP and GP conceived the study, participated in the study design and obtained funding. IZ, GP, AG and IMP participated in survey development and design of data collection. SV drafted the manuscript with input from all authors. All authors meet the International Committee of Medical Journal Editors criteria for authorship and have read and approved the final manuscript.

Funding The PRELUDE study is funded by the NSW Ministry of Health. Gilead Sciences is providing an in-kind supply of the study medication, Truvada. Gilead were not involved in the study or protocol design and will not have any role in data analysis. The views expressed in this publication do not necessarily represent the position of these organisations.

Competing interests SV, JM, DJT, MB, AM, JH, IMP and GP declare no competing interests. AG has received funding from bioCSL, ViiV and Gilead Sciences, and has also received conference travel funding and honoraria for educational presentations from Merck. IZ has received funding and in-kind support (supply of the study medication) from Gilead Sciences for this study.

Patient consent Obtained

Ethics approval St Vincent's Hospital Human Research Ethics Committee. Provenance and peer review Not commissioned; externally peer reviewed.

Open Access This is an Open Access article distributed in accordance with the Creative Commons Attribution Non Commercial (CC BY-NC 4.0) license, which permits others to distribute, remix, adapt, build upon this work noncommercially, and license their derivative works on different terms, provided the original work is properly cited and the use is non-commercial. See: http:// creativecommons.org/licenses/by-nc/4.0/

\section{REFERENCES}

1. UNAIDS. UNAIDS report on the global AIDS epidemic 2013. Geneva: Joint United Nations Programme on HIV/AIDS (UNAIDS), 2013.

2. The Kirby Institute. HIV, viral hepatitis and sexually transmissible infections in Australia. Annual Surveillance Report 2015. Sydney: Kirby Institute, 2015.

3. Baeten JM, Donnell D, Ndase P, et al. Antiretroviral prophylaxis for HIV prevention in heterosexual men and women. $N$ Engl J Med 2012;367:399-410.

4. Grant RM, Lama JR, Anderson PL, et al. Preexposure chemoprophylaxis for HIV prevention in men who have sex with men. N Engl J Med 2010;363:2587-99.

5. Choopanya K, Martin M, Suntharasamai $P$, et al. Antiretroviral prophylaxis for HIV infection in injecting drug users in Bangkok, Thailand (The Bangkok Tenofovir Study): a randomised, doubleblind, placebo-controlled phase 3 trial. Lancet 2013;381:2083-90.

6. Van Damme L, Corneli A, Ahmed K, et al. Preexposure prophylaxis for HIV infection among African women. $N$ Engl J Med 2012;367:411-22.

7. Corneli AL, Deese J, Wang M, et al. FEM-PrEP: adherence patterns and factors associated with adherence to a daily oral study product for pre-exposure prophylaxis. J Acquir Immune Defic Syndr 2014;66:324-31.

8. Anderson PL, Glidden DV, Liu A, et al. Emtricitabine-tenofovir concentrations and pre-exposure prophylaxis efficacy in men who have sex with men. Sci Transl Med 2012;4:151ra25.

9. Mccormack S, Dunn DT, Desai M, et al. Pre-exposure prophylaxis to prevent the acquisition of HIV-1 infection (PROUD): effectiveness results from the pilot phase of a pragmatic open-label randomised trial. Lancet 2016:387:53-60.
10. Molina JM, Capitant C, Spire B, et al. On-Demand Preexposure Prophylaxis in Men at High Risk for HIV-1 Infection. N Engl J Med 2015;373:2237-46.

11. Food and Drug Administration. FDA approves first drug for reducing the risk of sexually acquired HIV infection. Maryland: FDA, 2012 http://www.fda.gov/NewsEvents/Newsroom/PressAnnouncements/ ucm312210.htm (accessed 19 Nov 2015).

12. WHO. Guideline on when to start antiretroviral therapy and on pre-exposure prophylaxis for HIV. Geneva: World Health Organisation, 2015

13. Zablotska I. Pre-exposure prophylaxis of HIV with antiretroviral medications interim NSW guidelines. NSW PrEP Guidelines and clinical delivery group, 2014. http://www.cdc.gov/hiv/pdf/ prepguidelines2014.pdf (accessed 4 Apr 2016).

14. US Public Health Service. Preexposure prophylaxis for the prevention of HIV infection in the United States 2014- A clinica practice guideline. 2014.

15. ASHM. Australian commentary: US public health service clinical practice guidelines on prescribing PrEP. 2015. http://arv.ashm.org au/images/Australian_National_PrEP_Guidelines.PDF (accessed 4 Apr 2016)

16. Poynten IM, Jin F, Prestage GP, et al. Defining high HIV incidence subgroups of Australian homosexual men: implications for conducting HIV prevention trials in low HIV prevalence settings. HIV Med 2010;11:635-41.

17. Jin F, Prestage GP, Imrie J, et al. Anal sexually transmitted infections and risk of HIV infection in homosexual men. $J$ Acquir Immune Defic Syndr 2010;53:144-9.

18. Jin F, Prestage GP, Mcdonald A, et al. Trend in HIV incidence in a cohort of homosexual men in Sydney: data from the health in men study. Sex Health 2008;5:109-12.

19. Templeton DJ, Jin F, Mao L, et al. Circumcision and risk of HIV infection in Australian homosexual men. AIDS 2009;23:2347-51.

20. Cassell MM, Halperin DT, Shelton JD, et al. Risk compensation: the Achilles' heel of innovations in HIV prevention? BMJ 2006;332:605-7.

21. Marcus JL, Glidden DV, Mayer $\mathrm{KH}$, et al. No evidence of sexual risk compensation in the iPrEX trial of daily oral HIV preexposure prophylaxis. PLOS ONE 2013;8:e81997.

22. Kibengo FM, Ruzagira E, Katende D, et al. Safety, adherence and acceptability of intermittent tenofovir/emtricitabine as HIV pre-exposure prophylaxis (PrEP) among HIV-uninfected Ugandan volunteers living in HIV-serodiscordant relationships: a randomized, clinical trial. PLoS ONE 2013:8:e74314.

23. Volk JE, Marcus JL, Phengrasamy T, et al. No new HIV infections with increasing use of HIV preexposure prophylaxis in a clinical practice setting. Clin Infect Dis 2015;61:1601-3.

24. Liu AY, Vittinghoff E, Chillag K, et al. Sexual risk behavior among HIV-uninfected men who have sex with men participating in a tenofovir preexposure prophylaxis randomized trial in the United States. J Acquir Immune Defic Syndr 2013;64:87-94.

25. Liu AY, Cohen SE, Vittinghoff E, et al. Preexposure prophylaxis for HIV infection integrated with municipal- and community-based sexual health services. JAMA Intern Med 2016;176: 75-84.

26. ASHM. Antiretroviral guidelines: US DHHS guidelines with Australian commentary- when to start antiretroviral therapy in people with HIV. http://arv.ashm.org.au/clinical-guidance. (accessed 15 Oct 2015)

27. Scheibe A. Southern African guidelines for the safe use of pre-exposure prophylaxis in men who have sex with men who are at risk for HIV infection. SAJHIVMED 2012;13:15.

28. ACON. PrEP-Access options. 2015. http://endinghiv.org.au/nsw/ wp-content/uploads/2015/02/PrEP Access Options Paper1. pdf\#page =2 (accessed 19 Nov 2015).

29. Chen A, Dowdy DW. Clinical effectiveness and cost-effectiveness of HIV pre-exposure prophylaxis in men who have sex with men: risk calculators for real-world decision-making. PLOS ONE 2014;9: e108742.

30. Krakower DS, Jain S, Mayer KH. Antiretrovirals for primary HIV prevention: the current status of pre- and post-exposure prophylaxis. Curr HIVIAIDS Rep 2015;12:127-38.

31. Schneider K, Gray RT, Wilson DP. A cost-effectiveness analysis of HIV preexposure prophylaxis for men who have sex with men in Australia. Clin Infect Dis 2014;58:1027-34. 


\section{Correction: Protocol for an open-label, single-arm trial of HIV pre-exposure prophylaxis (PrEP) among people at high risk of HIV infection: the NSW Demonstration Project PRELUDE}

Vaccher S, Grulich A, McAllister J, et al. Protocol for an open-label, single-arm trial of HIV pre-exposure prophylaxis (PrEP) among people at high risk of HIV infection: the NSW Demonstration Project PRELUDE. BMJ Open 2016;6:e12179. The author affiliations are incorrect in this manuscript. Corrected affiliations are given below:

Authors

S Vaccher, ${ }^{1}$ A Grulich, ${ }^{1}$ J McAllister, ${ }^{2}$ D J Templeton, ${ }^{1,3}$ M Bloch, ${ }^{1,4}$ A McNulty, ${ }^{5}$ J Holden, ${ }^{6}$ I M Poynten, ${ }^{1}$ G Prestage, ${ }^{1}$ I Zablotska, ${ }^{1}$ on behalf of the PRELUDE Study Team

Author affiliations

${ }^{1}$ Kirby Institute, UNSW Australia, Kensington, New South Wales, Australia

${ }^{2}$ St Vincent's Hospital, Darlinghurst, New South Wales, Australia

${ }^{3}$ RPA Sexual Health, Sydney Local Health District, Camperdown, New South Wales, Australia

${ }^{4}$ Holdsworth House Medical Practice, Darlinghurst, New South Wales, Australia

${ }^{5}$ Sydney Sexual Health Centre, Sydney, New South Wales, Australia

${ }^{6}$ Centre for Population Health, NSW Ministry of Health, North Sydney, New South Wales, Australia

Open Access This is an Open Access article distributed in accordance with the Creative Commons Attribution Non Commercial (CC BY-NC 4.0) license, which permits others to distribute, remix, adapt, build upon this work noncommercially, and license their derivative works on different terms, provided the original work is properly cited and the use is non-commercial. See: http://creativecommons.org/licenses/by-nc/4.0/

BMJ Open 2016;6:e012179corr1. doi:10.1136/bmjopen-2016-012179corr1 